\title{
Controlled Release of 5-Fluorouracil from Adsorbents Containing Matrices Polymerized by Radiation
}

\author{
Masaru Yoshida, ${ }^{*}$ Minoru Kumakura, ${ }^{*}$, and Isao Kaetsu \\ Takasaki Radiation Chemistry Research Establishment, Japan Atomic Energy Research Institute, \\ Takasaki, Gunma 370-12, Japan.
}

(Received March 2, 1979)

\begin{abstract}
Entrapping of 5-fluorouracil (5-FU) was carried out by radiation-induced polymerization of glass-forming monomers such as 2-hydroxyethyl methacrylate (HEMA) and trimethylolpropane trimethacrylate (TMPT) at $-78 \mathrm{C}$ in the presence of adsorbents. It was found that the release of 5-FU from the matrix was retarded by the addition of various adsorbents. The release rate decreased with increasing concentrations of adsorbent. The manners of release of 5-FU from the matrices containing adsorbents were studied according to Higuchi's equation. The observation by a scanning electron microscope proved that an adsorbent was homogeneously distributed in the matrix.

KEY WORDS 5-Fluorouracil / Radiation-Induced Polymerization / Adsorbent / Glass-Forming Monomer / Controlled Release / 2-Hydroxyethyl Methacrylate / Trimethylolpropane Trimethacrylate /
\end{abstract}

The authors have been studying the characteristics of radiation-induced polymerization of glassforming monomers ${ }^{1}$ in the supercooled state and have found that these monomers have stable supercooling properties and large polymerizability at low temperatures. The applications of this lowtemperature polymerization to the immobilization of various biofunctional substances such as enzymes, ${ }^{2.3}$ microbial cells, ${ }^{4}$ organellas ${ }^{5}$ and drugs ${ }^{6.7}$ have been investigated. A detailed study on the controlled release of some drugs from matrices of various shapes such as flat-circular composites, microspheres, membranes, rods, and powders was also reported. ${ }^{8}$

It was shown in those studies that the release rate of drugs could be controlled by various methods, such as the increase of the hydrophilicity of the matrix, the addition of soluble polymer, and the addition of pore-making components. However, these methods were usually effective in promoting the release of drugs so as to increase the hydrophobic property of the matrix. In the present study, the matrix including 5-FU as a drug was prepared in the presence of various adsorbents and the effect of adsorbents on the retardation of drug release was studied.

\section{EXPERIMENTAL}

\section{Materials}

Glass-forming monomers such as 2-hydroxyethyl methacrylate (HEMA) and trimethylolpropane trimethacrylate (TMPT) were obtained from ShinNakamura Chemical Co., Ltd., and purified according to the methods described previously. ${ }^{6}$ Silica gel (230 - 400 mesh), activated charcoal (fine powder), molecular sieves 4A (40-60 mesh), and activated clay (fine powder) were the products of E. Merck AG., Nishio Industry Co., Ltd., and Kishida Chemical Co., Ltd., respectively. 5-Fluorouracil (5FU) as an anticancer was obtained from Nipponkayaku Co., Ltd., and pulverized to a powder (100-200 mesh).

\section{Preparation of Radiation Polymerized-Materices Containing 5-FU}

A mixture of $1.5 \mathrm{~cm}^{3}$ of glass-forming monomers, $100 \mathrm{mg}$ of $5-\mathrm{FU}$ and $0-300 \mathrm{mg}$ of adsorbents was charged into a glass ampoule of $8 \mathrm{~mm}$ inside diameter. The ampoule was degassed twice under cooling with liquid nitrogen and then was sealed under a vacuum of $10^{-3} \mathrm{mmHg}(0.13 \mathrm{~Pa})$. After shaking the ampoule, it was frozen at a fixed low 
temperature. The $\gamma$-ray irradiation from a ${ }^{60} \mathrm{Co}$ source was carried out for 2 hours at a dose rate of $5 \times 10^{5} \mathrm{R} / \mathrm{h}$ and at $-78^{\circ} \mathrm{C}$ in a Dewar vessel (dry ice-methanol). After irradiation, the hard composites $(8 \mathrm{~mm}$ diameter and $30 \mathrm{~mm}$ length) which formed were used for the dissolution test as in the polymerized state.

\section{Measurement of Release Rate}

The dissolution test to determine the release rate was carried out at $37^{\circ} \mathrm{C}$, while stirring at $100 \mathrm{rpm}$, with a Toyama Sangyo dissolution apparatus, Model TR-5S, based on United States Pharmacopeia XIX. In this work, the basket was immersed in $1000 \mathrm{~cm}^{3}$ of distilled water $(\mathrm{pH} 6.0)$ in the vessel. At a time interval over $16 \mathrm{~h}$ (in hydrophilic polymer system) or 30 days (in hydrophobic polymer system), a $5 \mathrm{~cm}^{3}$ sample of the dissolution medium was assayed spectrophotometrically, that is, with a Shimazu double beam spectrophotometer, Model UV-200, at $265 \mu \mathrm{m}$ for $5-\mathrm{FU}$.

\section{Electron Microscopic Observation}

The fine structure of the matrix was observed with a scanning electron microscope, Model JSM-U3 (Japan Electron Optics Laboratory Co., Ltd).

\section{RESULTS AND DISCUSSION}

Effect of Polymerization Conditions on Release of 5FU

Much of the inactivation of native 5-FU by irradiation can be avoided by choosing the conditions of lower temperatures than $0^{\circ} \mathrm{C}$ and smaller irradiation doses than $2 \times 10^{6} \mathrm{R}{ }^{8}$ Entrapping of 5FU can be carried out effectively under the above conditions using glass-forming monomers which form a stable supercooled state and polymerize quickly at low temperatures.

The time courses for the release of 5-FU from a hydrophilic matrix such as poly(2-hydroxyethyl methacrylate) (PHEMA) in the presence of adsorbents at $37^{\circ} \mathrm{C}$ are illustrated in Figures 1, 2, and 3 .

Figure 1 shows the time-release curve as a function of activated charcoal concentration added in the matrix. Figure 1 shows that the release of 5-FU was remarkably affected by the addition of activated charcoal and the release rate of 5-FU was retarded in the early stage of dissolution in the high concentration. The dissolution was later accelerated and

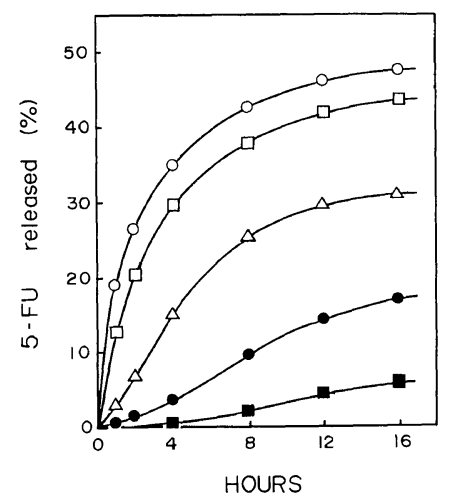

Figure 1. Drug release from PHEMA materix containing 5-FU as a function of activated charcoal concentration in HEMA drug adsorbent system. Activated charcoal concentration: $\bigcirc, 0 \mathrm{mg} ; \square, 10 \mathrm{mg} ; \triangle, 50 \mathrm{mg}$; $100 \mathrm{mg}$; $300 \mathrm{mg}$.

then suppressed again. The retarding effect caused by the adsorbent may be advantageous for the prolonged, durable release of drug from a large surface area matrix such as the finely divided one, from which the release is usually too quick. A similar tendency was observed in other adsorbents such as silica gel and molecular sieves 4A, as shown in Figure 2. However, the effect of adsorbent on 5-FU release differs greatly according to the kind of adsorbent. The retardation effect on the release was in the order of silica gel, molecular sieves $4 \mathrm{~A}$, activated charcoal, and clay. This can perhaps be attributed to differences in the adsorption properties of the drugs and in the physical structures of adsorbents inside the matrix. Figure 2 shows that the release rate of 5-FU was very large in the presence of activated clay, which is a nonadsorbent. Adsorption with an adsorbent expected a big influence on the retardation of drug release.

Figure 3 shows the rates of release in two samples obtained by different mixing methods in the HEMA drug activated charcoal system. According to this result, the release rate was larger in the case of a matrix prepared simultaneously than in that prepared stepwise. The following mechanism for the adsorbent effect on the release property might be supposed. Solid drug and adsorbent are first dispersed separately in the matrix. However, with the swelling of the polymer, the drug dissolves in water and then can be easily adsorbed by the adsorbent. After that, the desorption of the drug occurs in a 


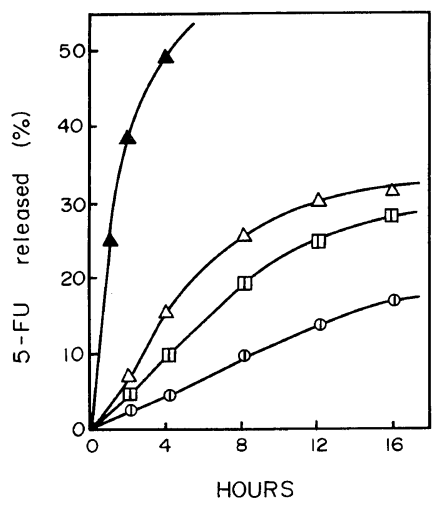

Figure 2. Drug release from PHEMA matrix containing 5-FU in the presence of various adsorbents in HEMA drug-adsorbent sytems. Adsorbent concentration, $50 \mathrm{mg}$. Adsorbent: $\boldsymbol{\Lambda}$, activated clay; $\triangle$, activated charcoal; $\square$, molecular sieves $4 \mathrm{~A}$; $($, silica gel.

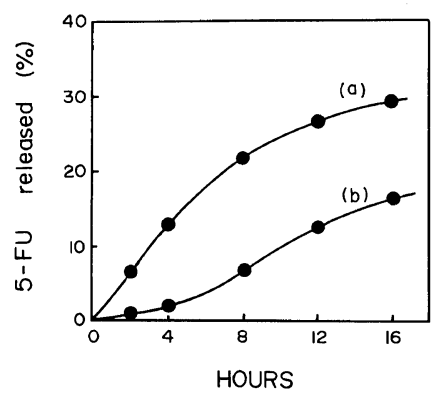

Figure 3. Effect of mixing method on release of 5-FU in HEMA drug activated charcoal system. Mixing method: (a) HEMA $\left(1.5 \mathrm{~cm}^{3}\right)$, activated charcoal $(50 \mathrm{mg})$, and 5 FU ( $30 \mathrm{mg}$ ) were mixed at the same time. (b) an aqueous solution (distilled water, $\left.\mathrm{pH} 6.0,2 \mathrm{~cm}^{3}\right)$ of 5 -FU $(30 \mathrm{mg}$ ) and activated charcoal $(50 \mathrm{mg})$ was mixed and the water was allowed to evaporate slowly under reduced pressure. Then, 5-FU adsorbed with activated charcoal was mixed with HEMA monomer $\left(1.5 \mathrm{~cm}^{3}\right)$.

certain equilibrium with the adsorption. The desorbed drug may be released out of the matrix by diffusion. Consequently, the release rate of drug in the presence of adsorbent decreases owing to such a adsorption-desorption process by the adsorbent, in comparison with that in the absence of adsorbent. Most of the drug would be released directly out of the matrix without being caught by adsorbent in the case of low adsorbent concentration, but the release of almost all the drug is retarded by the adsorption and the release becomes desorption-controlled in the system of high adsorbent concentration. This might be the reason for the different release behaviors in Figure 1 under various adsorbent concentrations. The swelling of polymer by water is almost completed within one hour and reaches a saturated state, so the swelling step is not a rate-determining process in the overall release in Figure 1.

The time course for the 5-FU release from a hard hydrophobic matrix, poly(trimethylolpropane trimethacrylate) (PTMPT), prepared by $\gamma$-irradiation in the presence of activated charcoal is shown in Figure 4. The release of 5-FU from this hard hydrophobic matrix was much greater than that from a soft hydrophilic matrix, in the presence of activated charcoal. This result shows that the hydrophobic property of the matrix also has an important effect on the drug release.

\section{Microscopic Observation of the Matrix}

The microphotographs of thee matrices obtained by $\gamma$-irradiation polymerization in the presence of adsorbents are shown in Figure 5. It was found that adsorbents are homogeneously distributed in the matrix in all systems, but fine structures in the matrices differed according to the kind of adsorbent. However, it seems that there are some differences in the density or vacancy of matrices in the presence of various adsorbents. That is, there are relatively many vacancies in the matrices such as activated clay and charcoal. While, there are few vacancies in the matrices such as silica gel and molecular sieves 4A. The larger release rates in the former two systems compared with the latter two cases (see Figure 2) might be roughly explained by such differences of apparent matrix density.

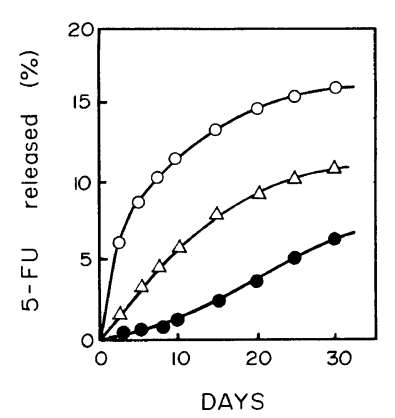

Figure 4. 5-FU release in PTMPT matrix as a function of activated charcoal concentration in TMPT drugadsorbent system. Activated charcoal concentration: $\bigcirc$, $0 \mathrm{mg} ; \triangle, 50 \mathrm{mg} ; 0,100 \mathrm{mg}$. 


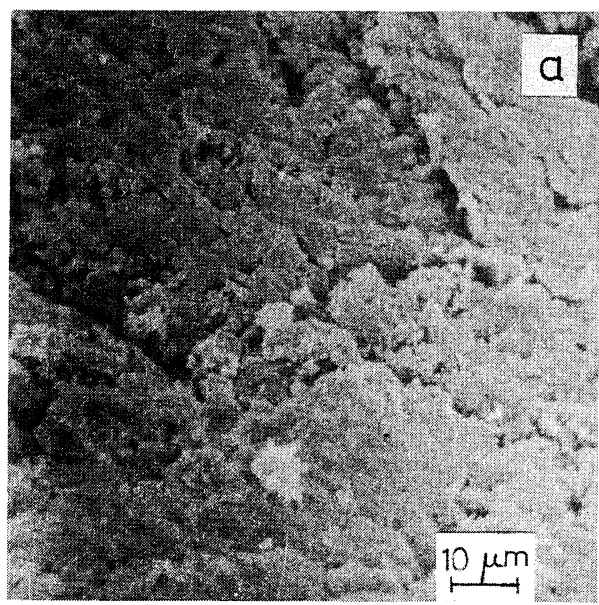

(a)

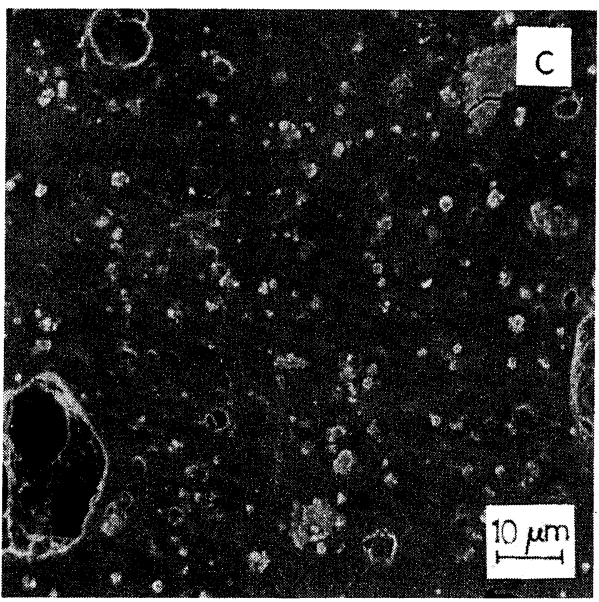

(c)

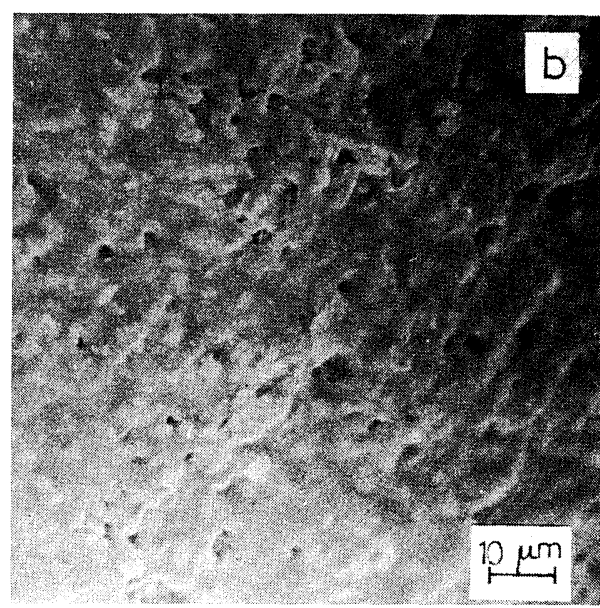

(b)

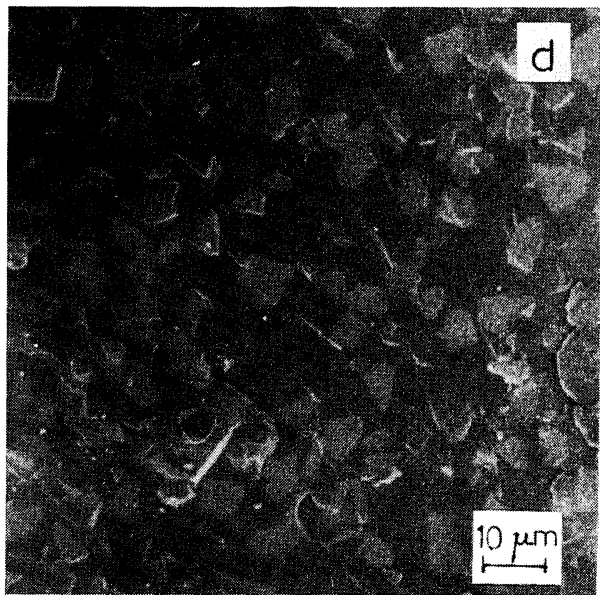

(d)

Figure 5. Scanning electron microphotographs of PHEMA matrices containing 5-FU in the presence of various adsorbents. Experimental conditions: the same as those in Figure 2. Adsorbent: (a), activated clay; (b), activated charcoal; (c), silica gel; (d), molecular sieves $4 \mathrm{~A}$.

\section{Kinetics of Release}

We reported previously that the release of drugs from radiation-polymerized materices, porous or nonporous, was carried out according to the Higuchi equation $^{9}$ for the diffusion-controlled release.

The 5-FU release from the present matrix including adsorbents was studied kinetically by the Higuchi equation. Assuming that in a quasi-steady state, the drug particle is small enough compared to the average distance of diffusion, and perfect sink conditions exist in the external media, the basic equation is

$$
Q=\left(2 A-C_{\mathrm{s}}\right) \sqrt{\frac{D t}{1+\frac{2\left(A-C_{\mathrm{s}}\right)}{C_{s}}}}
$$

where $Q$ is the amount of drug released after time $t$ per unit area of exposure, $A$ is the initial drug concentration, $C_{\mathrm{s}}$ is the solubility of drug in the external phase of the matrix, and $D$ is the diffusion constant of the drug molecule in the external phase of the matrix.

Figure 6 shows the relationship between the drug release and $t^{1 / 2}$ as a function of activated charcoal 


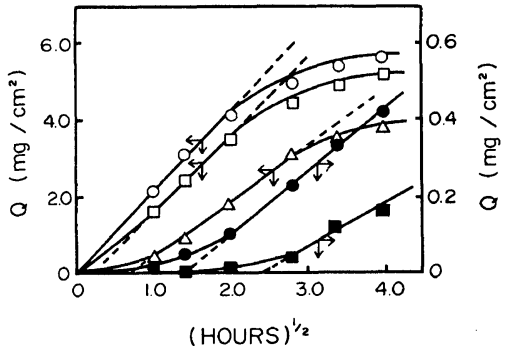

Figure 6. Linear $Q-t^{1 / 2}$ relationship in HEMA drug activated charcoal system. Experimental conditions and symbols are the same as those in Figure 1.

concentration, using the data given in Figure 1. Linear plots between $Q$ and $t^{1 / 2}$ were obtained over a certain time range for 5-FU from PHEMA matrix including activated charcoal, as seen in Figure 6. Furthermore, in including the system activated charcoal, extrapolation to the $\mathrm{x}$ axis indicates a lag time before sready state release. In matrix systems including no adsorbent, no time lag due to initial retardation was observed. The result in Figure 6 suggests that no drug is trapped on the matrix surface but rather the drug is trapped inside the matrix, outside or inside the adsorbent. That is, in those systems, the drug exists partly in a solid and partly in adsorbed state in adsorbent depending on the adsorbent concentration. These drugs are re- leased from the matrix phase directly by diffusion or stepwise by diffusion via desorption into the solution phase. Therefore, Higuchi's equation might be applicable over a certain equilibrium range, assuming that the apparent overall drug concentration consists of both markedly dispersed drug and adsorbeddesorbed drug.

Acknowledgement. The authors is grateful to Dr. $T$. Seguchi for his help with the electron microscope measurements.

\section{REFERENCES}

1. I. Kaetsu, H. Okubo, A. Ito, and K. Hayashi, J. Polymer Sci., A-1, 10, 2203 (1972).

2. I. Kaetsu, M. Kumakura, and M. Yoshida, Biotech. Bioeng., 21, 863 (1979).

3. M. Yoshida, M. Kumakura, and I. Kaetsu, Polymer, 20, 3 (1979)

4. M. Kumakura, M. Yoshida, and I. Kaetsu, J. SolidPhase Microbiology, 2, 279 (1978).

5. T. Fujimura, F. Yoshii, and I. Kaetsu, Plant Physiology, in press.

6. M. Yoshida, M. Kumakura, and I. Kaetsu, Polymer, 19, 1375 (1978).

7. M. Yoshida, M. Kumakura, and I. Kaetsu, J. Pharm Sci., 68, 860 (1979).

8. I. Kaetsu, M. Yoshida, and A. Yamada, J. Biomed. Mater. Res., in press.

9. T. Higuchi, J. Pharm. Sci., 50, 874 (1961). 